\title{
Fertility in luteinizing hormone receptor- knockout mice after wild-type ovary transplantation demonstrates redundancy of extragonadal luteinizing hormone action
}

\author{
Tomi Pakarainen,1 Fu-Ping Zhang,2 Matti Poutanen,1 and Ilpo Huhtaniemi1,3
} 1Department of Physiology, Institute of Biomedicine, University of Turku, Turku, Finland. ${ }^{2}$ Department of Physiology, Institute of Biomedicine, University of
Helsinki, Biomedicum Helsinki, Helsinki, Finland. ${ }^{3}$ Institute of Reproductive and Developmental Biology, Imperial College London, London, United Kingdom.

\begin{abstract}
The luteinizing hormone receptor (LHR), mainly expressed in gonads, is essential for normal reproduction. However, numerous recent studies have also demonstrated LHR expression in multiple extragonadal reproductive and nonreproductive tissues. Although some effects of luteinizing hormone (LH) or its agonist, human chorionic gonadotropin, have been shown in extragonadal sites, their physiological significance remains open. In the present study, we have addressed the function of the extragonadal LHR using LHR-KO mice (LuRKO mice), in which the ovaries of prepubertal mice were orthotopically replaced with pieces of WT ovary using similarly transplanted WT mice as controls . Most ovarian transplants attained normal endocrine function in both groups of mice, as demonstrated by normal age at vaginal opening, estrous cycles, and sexual behavior. Both the LuRKO and WT mice repeatedly became pregnant (9/16 vs. 16/20 after first mating; difference not significant) and delivered similarly sized litters, which grew normally after birth, indicating normal lactation. In conclusion, fertility is restored in LuRKO mice by transplantation of WT ovarian tissue. This is achieved in the absence of extragonadal LHR expression, which indicates physiological redundancy for such receptor sites.
\end{abstract}

\section{Introduction}

The regulatory functions of the 2 gonadotropins, luteinizing hormone (LH) and follicle-stimulating hormone (FSH), form the cornerstone of normal fertility. In addition to earlier studies on hypophysectomized animals, immunoneutralization of gonadotropins or gonadotropin-releasing hormone $(\mathrm{GnRH})$, and treatment with GnRH analogs, more recent studies on targeted inactivation of the gonadotropin subunit and receptor genes have specifically demonstrated the need of proper function of $\mathrm{LH}, \mathrm{FSH}$, and their receptors for the maintenance of female reproduction (1-7).

Quite unexpectedly, a number of studies have recently reported the presence of the common $\mathrm{LH}$ and human chorionic gonadotropin (hCG) receptor (LHR) in a variety of extragonadal tissues, including the uterus, oviduct, cervix, placenta, mammary gland, sperm, certain areas of the brain, and many others (8-11). Many of the findings were made on human tissues, but similar observations have been reported on a number of other mammalian species, including baboons, bovines, pigs, rats, and mice $(9,12-15)$. On the basis of these observations, a shift in the old paradigm that gonadotropins only have gonadal actions has been proposed (8). A number of extragonadal actions for LH/hCG have been suggested, including effects on brain development and sexual behavior, regulation of

Nonstandard abbreviations used: FSH, follicle-stimulating hormone; GnRH, gonadotropin-releasing hormone; hCG, human chorionic gonadotropin; LH, luteinizing hormone; LHR, common receptor for LH and hCG; LuRKO mouse, LH receptor-KO mouse; LuRKO-TR mouse, LuRKO mouse with transplanted WT ovaries; PRL, prolactin; TR mouse, transplanted mouse; WT-TR, WT mouse with transplanted WT ovaries.

Conflict of interest: The authors have declared that no conflict of interest exists.

Citation for this article: J. Clin. Invest. 115:1862-1868 (2005).

doi:10.1172/JCI24562. uterine blood flow, survival of early pregnancy, decidualization, and regulation of uterine growth and enzyme levels. However, although many of the findings on extragonadal LHR expression appear at face value convincing, there are several caveats to be considered regarding the concept of extragonadal gonadotropin (16). First, in several studies, only fragments of the LHR mRNA have been detected by RT-PCR analysis, without evidence for the presence of a fulllength message capable of encoding a functional receptor protein. Second, upon immunoblotting and immunohistochemistry, the size of the specific immunoreactive protein has often differed from that of the gonadal LHR, and often heterologous antisera without proper controls have been used. Third, although some rather convincing functional in vitro data exist, there is no compelling in vivo evidence for functionality of these receptors. In humans, the phenotype of inactivating LHR mutations seems to be limited to ovarian and testicular dysfunction, which speaks against a major role of the extragonadal LHR (17). Finally, all extragonadal phenotypes identified in our previous studies, both in LH receptor-KO (LuRKO) mice $(5,18)$ and in transgenic mice overproducing hCG $(19,20)$, can be explained solely by alterations of gonadal function.

To address the function of the extragonadal LHR in vivo, we have performed in the present study orthotopic ovarian transplantations on WT and LuRKO mice, and our data present strong direct evidence against the physiological significance of the extragonadal LH/hCG action.

\section{Results}

Ovarian transplantation. In total, $38 \mathrm{LuRKO}$ and $31 \mathrm{WT}$ females successfully underwent orthotopic ovarian transplantation. After operation, the occurrence of and age at vaginal opening and attainment of estrous cycle were observed as indicators of functional recovery 


\section{Table 1}

Age at vaginal opening, estrous cycle length, and number of ovulated oocytes in LuRKO-TR, WT-TR, and WT mice

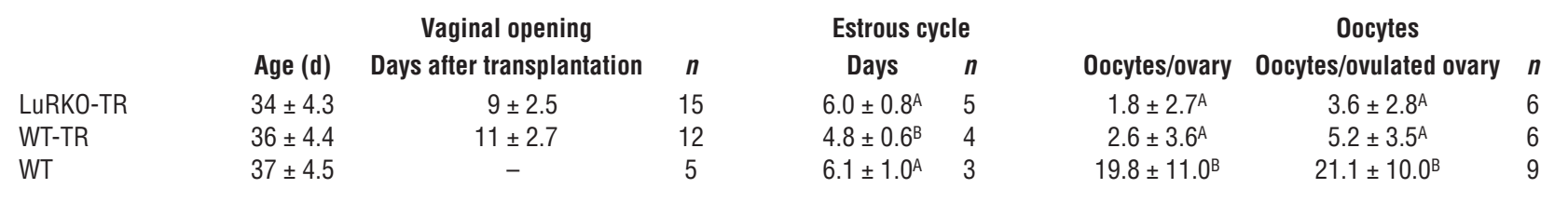

$n$, number of animals studied per each parameter; 2-4 estrous cycles were monitored per mouse. Oocytes/ovary, number of ovulated oocytes found in ampulla of all healthy-looking ovaries; oocyte/ovulated ovary, number of oocytes found in ampulla of the ovaries that had ovulated oocytes. Ovulations were stimulated by gonadotropins (see Methods). Superscript letters indicate significant differences between groups $(P<0.05)$. All data are expressed as mean \pm SD.

of the transplanted ovarian tissue. Twenty-seven LuRKO mice with transplanted WT ovaries (LuRKO-TR mice) (71\%) and 27 WT mice with transplanted WT ovaries (WT-TR mice) (87\%) showed normal uterine response, which indicated normal ovarian hormonal function of the transplanted tissue. Five of the LuRKO-TR mice presented with impaired estrous cycles and large, fluid-filled uteri. In 6 LuRKO-TR mice, the transplantation failed, and the mice presented upon autopsy with necrotic ovaries and failure of the uteri to respond to estradiol. For similar reasons, 4 of the WT transplantations were unsuccessful. Of the mice with apparently successful transplantation, 20 LuRKO-TR and 22 WT-TR females were randomly chosen for subsequent mating trials.

Vaginal opening, estrous cycle, and ovulatory capacity. Vaginal opening was used as an external indicator of estradiol response originating from vital and functional ovaries. The data indicated that vaginal opening occurred at very similar ages in LuRKO-TR, WT-TR, and WT mice $(34 \pm 4.3$ vs. $36 \pm 4.4$ vs. $37 \pm 4.5$ days, respectively; Table 1$)$, while no vaginal opening occurred in intact LuRKO mice. Furthermore, there was no difference in the length of estrous cycles of LuRKO-TR and WT mice ( $6.0 \pm 0.8$ vs. $6.1 \pm 1.0$ days, respectively), but in WT-TR mice, the length of the cycle was slightly shorter (4.8 \pm 0.6 days; $P<0.05$; Table 1$)$. However, in some mice of the transplanted (TR) groups, we detected impaired, irregular estrous cycles with a prolonged estrus. Later, these mice were observed to be infertile and to have large, fluid-filled uteri.

Analysis of the ovulatory capacity of the fertile mice indicated no differences between the transplanted groups, whereas in intact WT mice, a significantly larger amount of oocytes was detected after superovulation (Table 1 ). The TR mice also presented with anatomic abnormalities in their ovaries and oviducts, including adhesions, cysts, and missing ampullae of oviducts, when inspected under stereomicroscope. These findings clearly support the conclusion that the transplantation proce- dure itself explains, at least partly, their smaller litter sizes and lower fertility rate as compared with intact WT mice.

Breeding tests and weights of the offspring. Daily observations revealed that $95 \%$ of the WT-TR $(n=21)$ and $95 \%$ of LuRKO-TR mice $(n=19)$ analyzed mated, as indicated by the appearance of vaginal plugs, a sign of normal sexual behavior. All the intact WT mice $(n=10)$ were found to have vaginal plug. Following pregnancies of a normal length, 16 of 22 WT-TR mice (73\%) and 9 of 20 LuRKO-TR mice (45\%) gave birth to offspring. All 10 intact WT mice became pregnant. The somewhat higher pregnancy rate of the WT-TR mice did not differ significantly from that of the LuRKO-TR mice $(P<0.065)$. Nine originally fertile LuRKO-TR mice were subjected to further matings, and they became repeatedly pregnant, whereas the pregnancy rate of the 16 WT-TR mice, bred another 2 times, declined upon subsequent matings (Table 2). The litter sizes of the LuRKO-TR mice were not significantly smaller than those in the other study groups after the first breeding, and a significant difference existed only in the second and third pregnancies compared with intact WT and/or heterozygous control mice. The latter were used as an additional group of positive controls, as their fertility and litter sizes do not differ from those of WT mice. We subjected all infertile TR mice to autopsy in order to detect possible reasons for their infertility. In 4 LuRKO-TR females and in 2 WT-TR females, an anatomical cause was found for the infertility, such as regression of transplants, location of the transplanted tissue outside bursa ovary, or atrophied vaginal opening. Excluding mice in which the transplant was not technically successful, the fertility rates of the LuRKO-KO and WT-TR mice were re-calculated as $56 \%$ and $80 \%$, respectively.

After the first pregnancy, the litter size of LuRKO-TR females was $4.2 \pm 2.4$, which did not differ from that of the other groups studied (Table 2). The largest litter size $(n=9)$, found in a LuRKO-TR female, exceeded the average of all study groups. Weights of the pups on the day of delivery were equal, and their growth rates were

\section{Table 2}

Mating incidence of the first breeding and litter sizes following the first, second, and third pregnancies of LuRKO-TR, WT-TR, WT, and heterozygous mice

\begin{tabular}{|c|c|c|c|c|c|c|c|c|c|c|c|}
\hline & \multirow[b]{2}{*}{ Vaginal plug } & \multicolumn{4}{|c|}{ First pregnancy } & \multicolumn{3}{|c|}{ Second pregnancy } & \multicolumn{3}{|c|}{ Third pregnancy } \\
\hline & & First litter size & $n$ & \%/all & $\% /$ normal & Second litter size & $n$ & $\% /$ all & Third litter size & $n$ & $\% /$ all \\
\hline LuRKO-TR & $19 / 20(95 \%)$ & $4.2 \pm 2.4$ & 9 & $45 \%{ }^{A}$ & $56 \% \mathrm{~A}$ & $4.0 \pm 2.1^{\mathrm{A}}$ & 9 & $100 \%$ & $2.9 \pm 1.1^{\mathrm{A}}$ & 9 & $100 \%$ \\
\hline WT-TR & $21 / 22(95 \%)$ & $4.4 \pm 2.3$ & 16 & $73 \%{ }^{A}$ & $80 \%{ }^{A}$ & $5.2 \pm 3.0^{\mathrm{A}, \mathrm{B}}$ & 12 & $75 \%$ & $4.8 \pm 2.7^{\mathrm{A}, \mathrm{B}}$ & 8 & $67 \%$ \\
\hline WT & $10 / 10(100 \%)$ & $6.0 \pm 2.4$ & 10 & $100 \% \mathrm{~B}$ & $100 \% \mathrm{~B}$ & $7.9 \pm 2.2^{B}$ & 9 & $100 \%$ & $7.2 \pm 2.8^{B}$ & 9 & $100 \%$ \\
\hline $\mathrm{HZ}$ & - & $5.5 \pm 2.6$ & 52 & - & - & $5.6 \pm 2.6^{\mathrm{AB}}$ & 37 & - & $6.7 \pm 2.4^{\mathrm{B}}$ & 22 & - \\
\hline
\end{tabular}

$n$, number of litters born; \%/all, pregnancy rate of females mated; \%/normal, pregnancy rate of females with no obvious explanation for infertility. Superscript letters indicate significant differences between groups $(P<0.05)$. All data are expressed as mean \pm SD. - , not studied; HZ, heterozygous. 


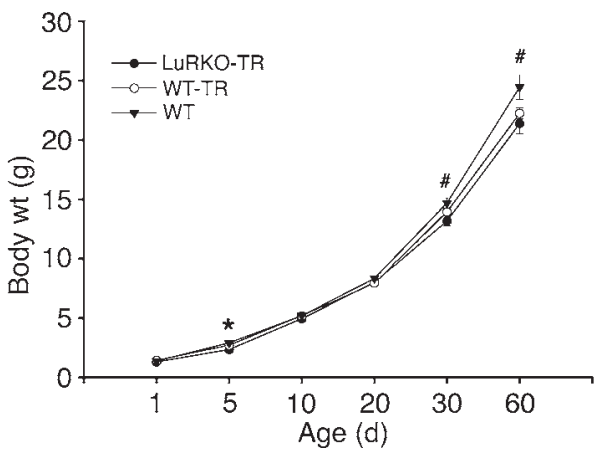

Figure 1

Body weights (mean \pm SEM) of the offspring of ovary-transplanted and control WT mice at the ages of $1,5,10,20,30$, and 60 days. ${ }^{*} P<0.05$, LuRKO-TR and WT-TR vs. WT; $P<0.05$, LuRKO-TR vs. WT. Each group consists of at least 4 litters; $n=16-58$ pups/age group.

similar in all study groups (Figure 1). Only at the ages of 5, 30, and 60 days, the offspring of the intact WT mice were slightly $(P<0.05)$ heavier than those of the mice with ovary transplants.

Body and organ weights and analysis of bursal size. At the time of transplantation (23 days of age), the body weights of recipient LuRKO and WT mice were similar (10.1 \pm 1.6 vs. $10.2 \pm 1.7$ g, respectively). However, already at this prepubertal age, a significant $55 \%$ reduction in uterine weights was observed in intact LuRKO mice $(3.2 \pm 0.91$ vs. $7.1 \pm 3.08 \mathrm{mg}$ in WT controls; $P<0.01)$. Likewise, the mean ovarian weights of intact LuRKO mice were $75 \%$ of those measured for WT mice $(1.5 \pm 0.28$ vs. $1.9 \pm 0.47 \mathrm{mg}$, respectively; $P<0.01)$ at the time of transplantation. The ovarian weights and the ovarian area, an index of the size of ovarian bursae, in LuRKO mice were similarly reduced compared with those of WT mice (Table 3).

There were no differences in body weights at the time of sacrifice (12-16 weeks) among the LuRKO-TR, WT-TR, and WT mice. Only the intact LuRKO mice were slightly but significantly heavier than the other groups (Table 4). The ovarian, uterine, pituitary, and adrenal gland weights of the LuRKO-TR, WT-TR, and WT mice were similar. Similar uterine weights of the LuRKO-TR mice, compared with WT-TR and intact WT mice, indicated normal estrogen response in LuRKO-TR mice. As expected, the weights of ovaries, uterus, and pituitary gland of the intact LuRKO mice were significantly lower than those of the other groups (Figure 2 and Table 4). The transplanted ovarian tissues responded well to endogenous LH stimulation in LuRKO-TR mice, and the growth of follicles from the resting primordial follicle pool normalized the ovarian weights (Figure 3). The ovarian weights in LuRKO-TR and WT-TR mice were slightly higher than those in WT mice, because with transplanted ovaries, it was difficult to remove all the connective tissue that adhered them to the peritoneal surface.
Ovarian and uterine histology and mammary gland whole-mount assessment. The histology of the WT ovaries successfully transplanted into LuRKO mice indicated the presence of follicles at all developmental stages, as well as of corpora lutea, while in LuRKO mice, follicular development was arrested at the antral stage (Figure 3). Histology of the uteri showed similar endometrial structure with tubular epithelium in LuRKO-TR, WT-TR, and WT mice. No differences were seen in the thickness or structure of the endometrial epithelium, stroma, or glands. In contrast, the uteri of intact LuRKO mice were small, the endometrium thin, the glandular structures sparse, and the epithelium lower than in the other groups (Figure 4). This difference is emphasized by the approximately 6- to 8-fold higher uterine weight in LuRKO-TR, WT-TR, and intact WT mice (Table 4). The uterine histology of the LuRKO-TR mice presenting with inappropriate estrous cycles indicated dysmorphic epithelium and abundance of polymorphonuclear leucocytes (data not shown).

Whole-mount analysis of the mammary glands indicated thin alveolar ducts with total absence of lobuli in the intact LuRKO mice. In contrast, the mammary glands of LuRKO-TR mice showed normal ducts and small terminal lobuli, similar to those observed in WT-TR and intact WT mice (Figure 5).

Hormone levels. The serum concentrations of $\mathrm{LH}$ and FSH, and those of pituitary prolactin (PRL), are presented in Figure 6. The LuRKO-TR females with successful ovarian transplantation showed normal negative feedback effect of ovarian endocrine activity on gonadotropin secretion, which resulted in marked reduction of $\mathrm{LH}$ in LuRKO-TR compared with intact LuRKO mice $(0.22 \pm 0.06 \mu \mathrm{g} / \mathrm{l}$ vs. $6.41 \pm 0.51 \mu \mathrm{g} / \mathrm{l})$. This concentration in LuRKO-TR was not significantly different from that in WT mice. The reduction in serum FSH levels after transplantations was smaller, remaining between the levels measured in intact WT and LuRKO mice. Pituitary PRL levels indicated total recovery in LuRKO-TR and WT-TR mice.

\section{Discussion}

Even though the gonads are the main target organ for LH and hCG, numerous studies have reported the presence of LHR in multiple non-gonadal tissues $(8,10)$. In the absence of in vivo data on their functionality, the physiological importance of these receptor sites has remained obscure. LuRKO mice are totally devoid of functional LHR, and this defect is reflected by severe underdevelopment of the reproductive organs due to the lack of gonadal sex steroid production (5). In the present study, we induced normal ovarian endocrine function and fertility in LuRKO mice by orthotopic transplantation of WT ovaries. Thus, in these mice, we were able to address in vivo the necessity of non-gonadal LHR for reproductive functions.

The ovarian transplantations successfully normalized the hypogonadal phenotype of the LuRKO females. The LuRKO mice with successfully transplanted WT ovaries demonstrated total recovery of reproductive functions, which indicates that the lack of adequate gonadal steroid synthesis was apparently the only reason for their

\section{Table 3}

Body and organ weights of the LuRKO and WT mice at the time of ovarian transplantation

\begin{tabular}{lccccccc} 
& $\boldsymbol{n}$ & Body wt $(\mathbf{g})$ & Age $(\mathbf{d})$ & $\boldsymbol{n}$ & Ovarian wt $(\mathbf{m g})$ & Ovarian area $\left(\mathbf{m m}^{2}\right)$ & Uterine wt $(\mathbf{m g})$ \\
LuRKO & 32 & $10.1 \pm 1.6$ & $22.8 \pm 1.8$ & 9 & $1.5 \pm 0.28^{\mathrm{A}}$ & $1.8 \pm 0.24^{\mathrm{A}}$ & $3.2 \pm 0.91^{\mathrm{A}}$ \\
WT & 22 & $10.2 \pm 1.7$ & $22.9 \pm 1.7$ & 9 & $1.9 \pm 0.47^{\mathrm{B}}$ & $2.1 \pm 0.36^{\mathrm{B}}$ & $7.1 \pm 3.08^{\mathrm{B}}$ \\
\hline
\end{tabular}

Ovarian area provides an index of the size of ovarian bursa. Superscript letters indicate significant differences between groups $(P<0.05)$. $n$, number of mice used for analyses. All data are expressed as mean \pm SD. 


\section{Table 4}

Body and organ weights of the LuRKO, LuRKO-TR, WT-TR, and WT mice at the age of $3-4$ months

\begin{tabular}{lcccc}
\hline & LuRKO & LuRKO-TR & WT-TR & WT \\
& $25.8 \pm 3.1^{\mathrm{A}}$ & $23.2 \pm 1.7^{\mathrm{B}}$ & $23.0 \pm 2.1^{\mathrm{B}}$ & $24.3 \pm 2.0^{\mathrm{A}, \mathrm{B}}$ \\
Body wt $(\mathrm{g})$ & $1.6 \pm 0.4^{\mathrm{A}}$ & $5.1 \pm 2.0^{\mathrm{B}}$ & $5.1 \pm 1.8^{\mathrm{B}}$ & $4.0 \pm 0.8^{\mathrm{B}}$ \\
Ovarian wt (mg) & $10.5 \pm 4.5^{\mathrm{A}}$ & $80.1 \pm 24.1^{\mathrm{B}}$ & $71.7 \pm 23.0^{\mathrm{B}}$ & $62.7 \pm 21.9^{\mathrm{B}}$ \\
Uterine wt (mg) & $2.8 \pm 0.68$ & $3.2 \pm 0.58$ & $3.1 \pm 0.47$ & $3.0 \pm 0.62$ \\
Adrenal gland wt (mg) & $1.4 \pm 0.29^{\mathrm{A}}$ & $2.0 \pm 0.35^{\mathrm{B}}$ & $1.8 \pm 0.41^{\mathrm{B}}$ & $1.7 \pm 0.41^{\mathrm{B}}$ \\
Pituitary gland wt (mg) & 10 & 15 & 10 & 11 \\
$n$ & & & & \\
\hline
\end{tabular}

$n$, number of animals/group used for analyses. Superscript letters indicate significant differences between groups $(P<0.05)$. All data are expressed as mean \pm SD. relatively long treatment would obviously be needed to achieve full uterine maturation from the severely atrophic state of the LHR-KO mice. Because our LuRKO-TR mice showed normal uterine histology and function, we trust that the complete physiological ovarian input, including sex steroids and other putative factors, is needed to restore the uterine phenotype, and this can be achieved in the absence of uterine LHR. Although we did not quantify the cell components of the uterus, the functional data, i.e., occurrence of pregnancies and normal histological appearance, provide strong evidence that the uteri were sufficiently mature to maintain normal pregnancy in the absence of LHR expression.

extragonadal phenotype and that their infertility was caused by the arrest of folliculogenesis and anovulation in the absence of LH signaling. The success rate of ovarian transplantations was somewhat, though not significantly, lower in LuRKO than in WT recipients. We assume that this reflects the greater technical difficulty of performing transplantation on the LuRKO mice because of the underdevelopment of their reproductive organs. This was supported by the findings that already prior to puberty, at the age of transplantation, the uterine weights and sizes of bursae ovari of the LuRKO females were reduced compared with those of WT mice. These findings may also indicate poorer vascularization of the bursae and ovaries in LuRKO females, which would reduce the vitality of transplants. The postnatal levels of gonadotropins are high in female mice (21), and there is evidence for the onset of ovarian sex steroid production already during the first week of life (22). This provides an explanation for the higher ovarian and uterine weights of WT compared with LuRKO mice at the age of transplantation.

Ovarian transplantation fully recovered the macroscopic phenotypes of LuRKO females, and all the organ weights measured were in the range of those in WT mice. Furthermore, serum gonadotropin levels showed normal negative feedback response to normalized ovarian function. However, abnormal estrous cycles with prolonged estrus and enlarged uteri were more frequent in LuRKO-TR mice. This was expected due to their abnormal hypothalamic-pituitary-gonadal function before puberty. Despite the apparently normal ovarian function, serum FSH levels remained slightly (but not significantly) elevated in the LuRKO-TR mice. Therefore, the disruption of the LHR signaling postnatally may have some longterm effects that do not totally recover after ovarian transplantation at puberty. A similar finding was previously reported from an ovarian transplantation study using aromatase-KO mice in which the fertility rate only reached $30 \%(23)$.

The current data demonstrated a recovery of the atrophic uteri and fertility of LuRKO-TR mice. In a previous study, Lei et al. $(6,24)$ subjected another KO mouse model for LHR (LHR-KO) to estrogen and progesterone replacement therapy between 30 and 52 days of life. The treatment induced vaginal opening but did not affect ovarian morphology. The uterus became thicker, but the number of endometrial glands remained low, and the animals remained infertile. Persistent anovulation was the apparent reason for infertility, but the incomplete reversal of the uterine phenotype was interpreted by the authors as a sign of direct $\mathrm{LH}$ action on this organ. However, it can be argued that it is difficult to mimic by exogenous hormone treatment the ovarian steroidogenesis that is needed to induce full functional response of the uterus. Furthermore, a
Upon mating, both LuRKO-TR and WT-TR mice displayed normal female sexual behavior, as indicated by the detection of vaginal plugs following successful mating. There is considerable evidence that $L H R$ is expressed in developing and adult nervous tissue. mRNA transcripts for $L H R$ have been shown in the brain tissue of rats, bovines, and humans (25-28). Reporter genes driven by various lengths of the $L H R$ promoter have been found to be expressed in the brain of transgenic mice $(29,30)$. Furthermore, Apaja and colleagues (15) recently showed endogenous LHR mRNA, protein, and ligand binding in various rat brain areas both during embryogenesis and in adult age. Specifically, LHR promoter activity was found in the developing olfactory bulb, sensory and autonomic ganglia, thalamus, hypothalamus, and brain stem and additionally in adult cerebral cortex, cerebellum, and posterior hippocampus. The authors hypothesized that LHR may play a role in neuronal growth and differentiation and, in adult age, in reproductive behavior, memory, and motor coordination. Although we did not address these functions specifically in the LuRKO-TR mice, their reproductive behavior upon mating was indistinguishable from that of the WT controls. Hence, if the neural structures regulating reproductive functions are under direct by other effectors in the LHR-deficient situation.

The fertility of the LuRKO-TR mice, as indicated by successful delivery of normal-sized litters, was somewhat, though not significantly, reduced in LuRKO-TR (56\%) compared with WT-TR mice (80\%), while the litter sizes at the first pregnancy were similar in both ovary-transplanted groups and WT controls. A lower fertility rate in LuRKO-TR mice could be expected in view of the fact that they were not exposed to normal levels of ovarian
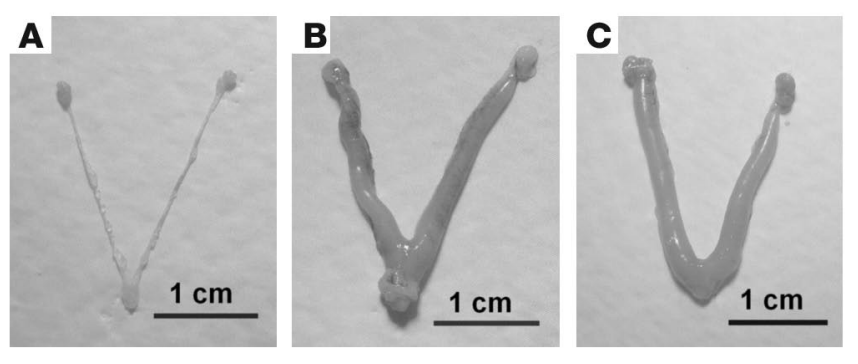

\section{Figure 2}

Representative macroscopic images of the uteri and ovaries of LuRKO (A), LuRKO-TR (B), and WT (C) mice. The slight hyperemia in the LuRKO-TR uterus was not a consistent finding. LH/hCG action, such effects are only minor and compensated for 


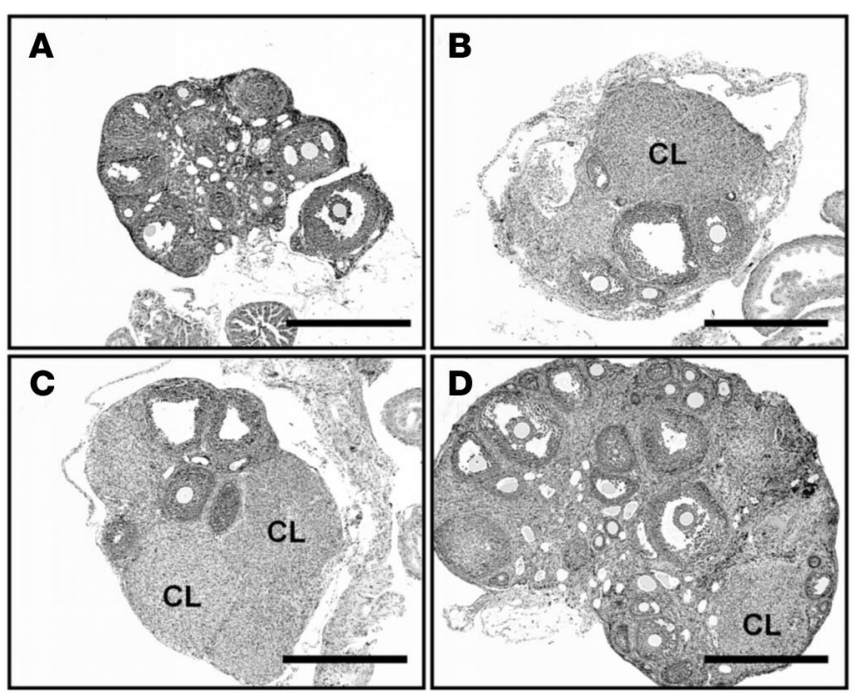

Figure 3

Representative light microscopic images of the ovaries of LuRKO (A), LuRKO-TR (B), WT-TR (C), and WT (D) mice. CL, corpus luteum. Scale bars, $500 \mu \mathrm{m}$.

steroids during the first 3 weeks post partum preceding the transplantation. This period represents in rodents an important priming and growth period of the female reproductive organs (21), and if it is delayed, the recovery after ovarian transplantation may not be total. Another reason may be that ovarian transplantation is technically more challenging in the hypogonadal LuRKO mice. The finding that all the fertile LuRKO-TR mice were able to maintain their fertility reflects the fact that reasons other than uterine dysfunction were behind their slightly lower fertility rate. The analysis of ovulatory capacity demonstrated a significantly diminished number of oocytes in TR mice after superovulation. Stereomicroscopic inspection also revealed anatomical alterations contributing to the lower fertility rates and litter sizes of the TR groups. The LuRKO-TR mice were evidently more affected by the surgery than their WT littermates, as their ovarian bursae were significantly smaller and more fragile at the time of transplantation. Nevertheless, we provide here strong evidence that LuRKO mice transplanted with WT ovaries can attain fertility at a rate that is not significantly different from that of identically transplanted WT littermates.

Our findings speak strongly against the physiological significance of extragonadal LHR expression in female mice. However, there is a large body of evidence for the presence of such receptors in various extragonadal organs $(8-10)$. Although the level of LHR expression in the extragonadal sites is generally low (15, $31,32)$ and functional data are sparse, there are some rather convincing in vitro findings of hCG-stimulated signal transduction and biochemical responses in non-gonadal sites, in particular in the uterus. The signaling involved in these actions invariably differs from the cAMP-mediated action of LH in gonads (11, 33-35). They may represent another example of the kind of ectopic receptor expression that is common with $G$ protein-coupled receptors; for instance, the $\mathrm{GnRH}$ receptor in various malignancies (36) and the gastrointestinal polypeptide receptor in nodular adrenal hyperplasia and in Cushing syndrome (37-39). The extragonadal LHRs may respond to pharmacological doses of
LH/hCG and offer a target for pharmacotherapy, but their physiological role as regulators of development and maintenance of reproductive functions seems unlikely.

In conclusion, the LuRKO mice presented after orthotopic transplantation of WT ovaries with largely normal sexual behavior, fertility, and lactation compared with similarly transplanted WT controls. It is prudent to state that extragonadal LHR expression is not a prerequisite for normal fertility of female mice. With respect to human fertility, this question remains open.

\section{Methods}

Animals. The LuRKO mice were created by targeted disruption of exon 11 of the LHR, and the mutant mouse line produced was maintained by inbreeding of these C57BL/6J-129/SvJ hybrids. The basic characteristics of the reproductive phenotype of LuRKO mice have been previously described $(5,18)$. The animals were housed under controlled environmental conditions (12-hour light/dark cycle; temperature $21 \pm 1^{\circ} \mathrm{C}$ ) in the animal facility of the University of Turku. They were fed mouse chow SDS RM-3 (Special Diets Services) and tap water ad libitum. We genotyped mice using the PCR method described previously (5). All procedures were approved by the University of Turku Lab-Animal Care and Use Committee.

Orthotopic ovary transplantation. At an average age of 23 days, 38 LuRKO and 31 WT mice $(22.8 \pm 1.8$ vs. $22.9 \pm 1.7$ days old, respectively) were anesthetized with $100-300 \mu \mathrm{l}$ of $2 \%$ tribromoethanol (Avertin; SigmaAldrich; ref. 40), and buprenorphine (Temgesic, 3-5 $\mu \mathrm{g} /$ mouse s.c.; Schering-Plough) was used for postoperative analgesia. The method used for transplantation has been described previously (41). In short, both ovaries were removed surgically from the bursa surrounding ovaries and each was

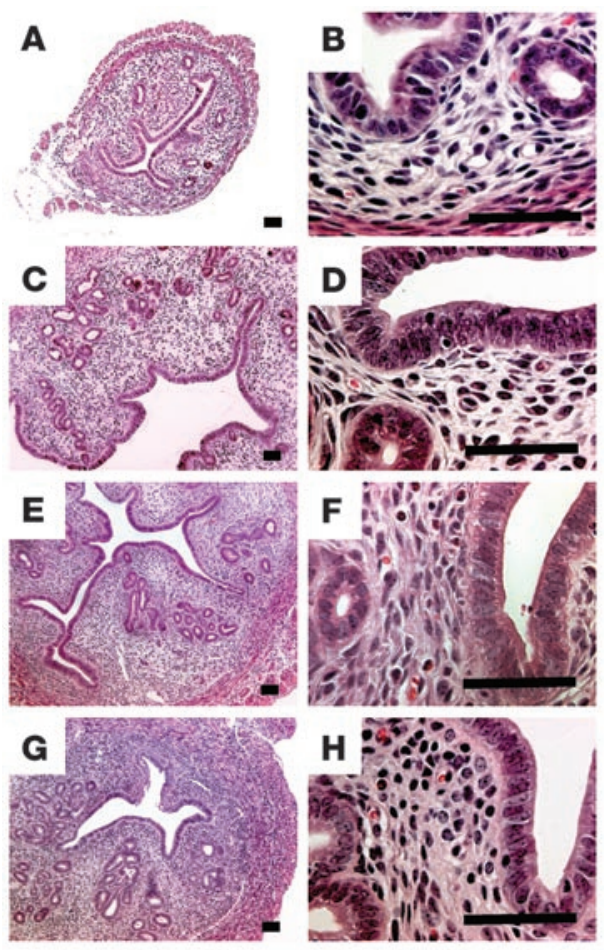

Figure 4

Representative light microscopic images of uterine histology in intact LuRKO (A and B), LuRKO-TR (C and D), WT-TR (E and F), and intact WT $(\mathbf{G}$ and $\mathbf{H})$ mice. The images on the right are at higher magnification. Scale bars, $50 \mu \mathrm{m}$. 


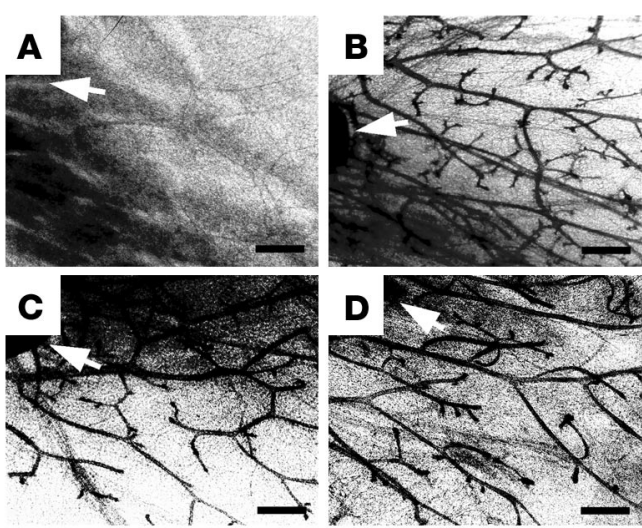

Figure 5

Representative whole mounts of mammary glands of LuRKO (A), LuRKO-TR (B), WT-TR (C), and WT (D) mice. Arrows indicate the position of a lymph node. All the images are at the same magnification. Scale bars, $500 \mu \mathrm{m}$.

replaced with one-third to one-half of an intact ovary of a $22.9 \pm 1.7$-dayold WT mouse, and skin was sutured after the operation. The operation was also carried out on WT mice, with their ovaries replaced with a piece of WT littermate ovary. These mice served as methodological controls for the success of the operation. Intact LuRKO and WT mice were used as negative and positive fertility controls.

Measurement of ovarian and uterine weights and assessment of size of the ovarian bursa. A total of 9 LuRKO and 10 WT mice were sacrificed at the age of ovarian transplantation. Body, ovarian, and uterine weights were measured. To assess the size of the ovarian bursa, we photographed the largest side of the ovary under stereomicroscope, and the area of the bursa tightly surrounding the ovaries was measured from the pictures using Leica IM1000 software (Leica Inc.; Leica Microsystems).

Assessment of vaginal opening and estrous cycle. After ovarian transplantations, the mice were inspected 3 times a week for vaginal opening, used as indicator of regained endocrine function of the ovarian transplants. After occurrence of vaginal opening, vaginal epithelial cell smears were used to assess the phase of estrous cycle. This was done by washing the vaginal epithelium with 1 drop of PBS and transferring the washing solution on a microscopic slide and then drying it in air. The cells were fixed for a few seconds in $96 \%$ and $100 \%$ ethanol and stained with Mayer's hematoxylin. After staining, the specimens were mounted with Aquamount (BDH-Gurr) and analyzed under a light microscope. For analysis of the length of estrous cycle, the vaginal samples were taken daily for 17 consecutive days, starting at the age of 2 months.

Analysis of ovulatory capacity. Six to 9 fertile LuRKO-TR, WT-TR, and intact WT mice were stimulated for ovulation by i.p. injection of $10 \mathrm{IU}$ of pregnant mare serum gonadotropin (PMSG; Sigma-Aldrich) and 48 hours later by 5 IU of hCG (Organon). Twenty-four hours later, the mice were sacrificed, and oviducts were placed into flushing holding medium (FHM; Specialty Media). After the cumulus-oocyte cell mass was dispersed by hyaluronidase treatment, the number of oocytes found in ampulla of the oviduct was calculated under stereomicroscope.

Breeding tests. A randomly selected group of 20 LuRKO-TR and 22 WT-TR female mice were bred with WT males of proven fertility at the age of 7-9 weeks. Each female was placed separately in a cage together with a male, and vaginal plugs were inspected daily. When a pregnancy was observed, the male was removed from the cage before delivery. The mice not conceiving within 1-2 months' breeding were considered infertile.

After delivery, the number and sex of the offspring were determined, and the litters were inspected daily. The ability of the females to nurse their offspring was monitored, and the weights of pups from at least 4 litters per study group were measured at 1, 5, 10,20,30, and 60 days of age. After weaning at 20 days, 9 LuRKO-TR and 16 WT-TR females were bred again so that the persistence of their fertility and the litter sizes of a second and third pregnancy could be analyzed. Upon later breedings, the females were mated with a different male from that in the first breeding test.

Specimen collection. At the age of 12 to 16 weeks, transplanted virgin females were sacrificed and samples collected. All mice were killed at the diestrous phase of the cycle, as indicated by vaginal epithelial cytology. The mice were anesthetized with $300-500 \mu l$ of $2 \%$ Avertin and weighed, and blood was collected by cardiac puncture. The uterus, ovaries, and adrenal and pituitary glands were weighed and either fixed for histological analyses or snap-frozen in liquid nitrogen. The inguinal mammary glands were fixed for whole-mount analyses. Frozen samples were stored at $-70^{\circ} \mathrm{C}$.

Histological analyses and mammary gland whole mounts. The ovaries, uteri, and pieces of mammary gland were fixed overnight in $4 \%$ paraformaldehyde, dehydrated, and embedded in paraffin. Tissues were sectioned at $5-\mu \mathrm{m}$ thickness and stained with H\&E. Inguinal mammary glands for wholemount analyses were spread on a glass slide and fixed overnight in $25 \%$ acetic acid in $75 \%$ ethanol. The samples were thereafter washed in $70 \%$ ethanol in water and stained overnight in carmine (Sigma-Aldrich), dehydrated in ethanol and xylene, and mounted with coverslips.

Hormone measurements. We analyzed the serum concentrations of LH and FSH using immunofluorometric assays as described previously $(42,43)$.
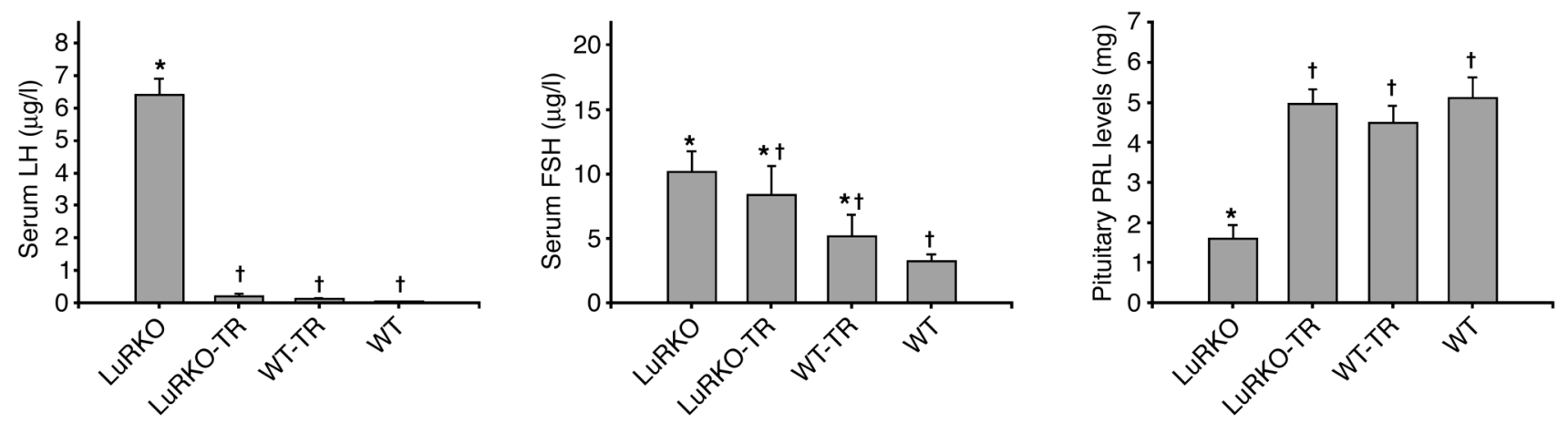

Figure 6

Serum LH and FSH concentrations and pituitary PRL levels in LuRKO, LuRKO-TR, WT-TR, and WT mice. $n=8-10$ mice/group; data are expressed as mean \pm SEM. The asterisks and crosses indicate significant differences between groups $(P<0.05)$. 
PRL contents of the pituitary homogenates were measured by RIA, as described previously (44).

Statistical analyses. SigmaStat software (SPSS Inc.) was used for statistical analyses. ANOVA (multiple comparisons) Student's $t$ test (2 groups), and Fisher exact test were used. After ANOVA, Dunn and Student-Newman-Keuls post-hoc tests were used to identify the groups differing. The significance was set at $P<0.05$, and the values were presented as mean \pm SD or SEM, as specified.

\section{Acknowledgments}

We wish to thank Nina Messner, Tarja Laiho, Johanna Lahtinen, and Hannele Rekola for excellent technical assistance. This study was supported by a Centre of Excellence Grant from the Academy of Finland (to I. Huhtaniemi and M. Poutanen), a Programme Grant from the Wellcome Trust (to I. Huhtaniemi), and the Research and Science Foundation of Farmos (to T. Pakarainen).

Received for publication January 25, 2005, and accepted in revised form April 26, 2005.

Address correspondence to: Ilpo Huhtaniemi, Institute of Reproductive and Developmental Biology, Imperial College London, Du Cane Road, London W12 0NN, United Kingdom. Phone: 44-20-7594-2104; Fax: 44-20-7594-2184; E-mail: ilpo.huhtaniemi@imperial.ac.uk.
1. Kendall, S.K., Samuelson, L.C., Saunders, T.L., Wood, R.I., and Camper, S.A. 1995. Targeted disruption of the pituitary glycoprotein hormone alpha-subunit produces hypogonadal and hypothyroid mice. Genes Dev. 9:2007-2019.

2. Kumar, T.R., Wang, Y., Lu, N., and Matzuk, M.M. 1997. Follicle stimulating hormone is required for ovarian follicle maturation but not male fertility. Nat. Genet. 15:201-204.

3. Dierich, A., et al. 1998. Impairing follicle-stimulating hormone (FSH) signaling in vivo: targeted disruption of the FSH receptor leads to aberrant gametogenesis and hormonal imbalance. Proc. Natl. Acad. Sci. U. S. A. 95:13612-13617.

4. Abel, M.H., et al. 2000. The effect of a null mutation in the follicle-stimulating hormone receptor gene on mouse reproduction. Endocrinology 141:1795-1803.

5. Zhang, F.P., Poutanen, M., Wilbertz, J., and Huhtaniemi, I. 2001. Normal prenatal but arrested postnatal sexual development of luteinizing hormone receptor knockout (LuRKO) mice. Mol. Endocrinol. 15:172-183.

6. Lei, Z.M., et al. 2001. Targeted disruption of luteinizing hormone/human chorionic gonadotropin receptor gene. Mol. Endocrinol. 15:184-200.

7. Ma, X., Dong, Y., Matzuk, M.M., and Kumar, T.R. 2004. Targeted disruption of luteinizing hormone beta-subunit leads to hypogonadism, defects in gonadal steroidogenesis, and infertility. Proc. Natl. Acad. Sci. U. S. A. 101:17294-17299.

8. Rao, C.V. 2001. Multiple novel roles of luteinizing hormone. Fertil. Steril. 76:1097-1100.

9. Zheng, M., Shi, H., Segaloff, D.L., and Van Voorhis, B.J. 2001. Expression and localization of luteinizing hormone receptor in the female mouse reproductive tract. Biol. Reprod. 64:179-187.

10. Fields, M.J., and Shemesh, M. 2004. Extragonadal luteinizing hormone receptors in the reproductive tract of domestic animals. Biol. Reprod. 71:1412-1418.

11. Shemesh, M. 2001. Actions of gonadotrophins on the uterus. Reproduction. 121:835-842.

12. Ziecik, A.J., Stanchev, P.D., and Tilton, J.E. 1986. Evidence for the presence of luteinizing hormone/ human chorionic gonadotropin-binding sites in the porcine uterus. Endocrinology. 119:1159-1163.

13. Fazleabas, A.T., Donnelly, K.M., Srinivasan, S., Fortman, J.D., and Miller, J.B. 1999. Modulation of the baboon (Papio anubis) uterine endometrium by chorionic gonadotrophin during the period of uterine receptivity. Proc. Natl. Acad. Sci. U. S. A. 96:2543-2548.

14. Shemesh, M., et al. 2001. Functional importance of bovine myometrial and vascular $\mathrm{LH}$ receptors and cervical FSH receptors. Semin. Reprod. Med. 19:87-96.

15. Apaja, P.M., Harju, K.T., Aatsinki, J.T., Petaja-Repo, U.E., and Rajaniemi, H.J. 2004. Identification and structural characterization of the neuronal luteinizing hormone receptor associated with sensory systems. J. Biol. Chem. 279:1899-1906.
16. Stewart, E.A. 2001. Gonadotropins and the uterus: is there a gonad-independent pathway? J. Soc. Gynecol. Investig. 8:319-326.

17. Themmen, A.P.N., and Huhtaniemi, I.T. 2000. Mutations of gonadotropins and gonadotropin receptors: elucidating the physiology and pathophysiology of pituitary-gonadal function. Endocr. Rev. 21:551-583.

18. Zhang, F.P., Pakarainen, T., Poutanen, M., Toppari, J., and Huhtaniemi, I. 2003. The low gonadotropinindependent constitutive production of testicular testosterone is sufficient to maintain spermatogenesis. Proc. Natl. Acad. Sci. U. S. A. 100:13692-13697.

19. Rulli, S.B., et al. 2002. Reproductive disturbances, pituitary lactotrope adenomas, and mammary gland tumors in transgenic female mice producing high levels of human chorionic gonadotropin. Endocrinology. 143:4084-4095.

20. Rulli, S.B., et al. 2003. Elevated steroidogenesis, defective reproductive organs, and infertility in transgenic male mice overexpressing human chorionic gonadotropin. Endocrinology. 144:4980-4990.

21. Halpin, D.M., Jones, A., Fink, G., and Charlton, H.M. 1986. Postnatal ovarian follicle development in hypogonadal (hpg) and normal mice and associated changes in the hypothalamic-pituitary ovarian axis. J. Reprod. Fertil. 77:287-296.

22. Gray, S.A., Mannan, M.A., and O'Shaughnessy, P.J. 1995. Development of cytochrome P450 aromatase mRNA levels and enzyme activity in ovaries of normal and hypogonadal (hpg) mice. J. Mol. Endocrinol. 14:295-301.

23. Toda, K., et al. 2004. Aromatase-knockout mouse carrying an estrogen-inducible enhanced green fluorescent protein gene facilitates detection of estrogen actions in vivo. Endocrinology. 145:1880-1888.

24. Rao, C.V., and Lei, Z.M. 2002. Consequences of targeted inactivation of $\mathrm{LH}$ receptors. Mol. Cell. Endocrinol. 187:57-67.

25. Lei, Z.M., Rao, C.V., Kornyei, J.L., Licht, P., and Hiatt, E.S. 1993. Novel expression of human chorionic gonadotropin/luteinizing hormone receptor gene in brain. Endocrinology. 132:2262-2270.

26. Lei, Z.M., and Rao, C.V. 2001. Neural actions of luteinizing hormone and human chorionic gonadotropin. Semin. Reprod. Med. 19:103-109.

27. al-Hader, A.A., Lei, Z.M., and Rao, C.V. 1997. Neurons from fetal rat brains contain functional luteinizing hormone/chorionic gonadotropin receptors. Biol. Reprod. 56:1071-1076.

28. al-Hader, A.A., Lei, Z.M., and Rao, C.V. 1997. Novel expression of functional luteinizing hormone/ chorionic gonadotropin receptors in cultured glial cells from neonatal rat brains. Biol. Reprod. 56:501-507.

29. Hamalainen, T., Poutanen, M., and Huhtaniemi, I. 1999. Age- and sex-specific promoter function of a 2-kilobase 5 '-flanking sequence of the murine luteinizing hormone receptor gene in transgenic mice. Endocrinology. 140:5322-5329.

30. Hamalainen, T., Poutanen, M., and Huhtaniemi, I.
2001. Promoter function of different lengths of the murine luteinizing hormone receptor gene 5 'flanking region in transfected gonadal cells and in transgenic mice. Endocrinology. 142:2427-2434.

31. Lei, Z.M., Toth, P., Rao, C.V., and Pridham, D. 1993. Novel coexpression of human chorionic gonadotropin (hCG)/human luteinizing hormone receptors and their ligand hCG in human fallopian tubes. J. Clin. Endocrinol. Metab. 77:863-872.

32. Tao, Y.X., Lei, Z.M., Woodworth, S.H., and Rao, C.V. 1995. Novel expression of luteinizing hormone/chorionic gonadotropin receptor gene in rat prostates. Mol. Cell. Endocrinol. 111:R9-R12.

33. Srisuparp, S., et al. 2003. Signal transduction pathways activated by chorionic gonadotropin in the primate endometrial epithelial cells. Biol. Reprod. 68:457-464.

34. Eta, E., Ambrus, G., and Rao, C.V. 1994. Direct regulation of human myometrial contractions by human chorionic gonadotropin. J. Clin. Endocrinol. Metab. 79:1582-1586.

35. Zygmunt, M., et al. 2002. Characterization of human chorionic gonadotropin as a novel angiogenic factor. J. Clin. Endocrinol. Metab. 87:5290-5296.

36. Harrison, G.S., Wierman, M.E., Nett, T.M., and Glode, L.M. 2004. Gonadotropin-releasing hormone and its receptor in normal and malignant cells. Endocr. Relat. Cancer. 11:725-748.

37. Lacroix, A., et al. 1992. Gastric inhibitory polypeptidedependent cortisol hypersecretion - a new cause of Cushing's syndrome. N. Engl. J. Med. 327:974-980.

38. Lebrethon, M.C., et al. 1998. Food-dependent Cushing's syndrome: characterization and functional role of gastric inhibitory polypeptide receptor in the adrenals of three patients. J. Clin. Endocrinol. Metab. 83:4514-4519.

39. Groussin, L., et al. 2002. The ectopic expression of the gastric inhibitory polypeptide receptor is frequent in adrenocorticotropin-independent bilateral macronodular adrenal hyperplasia, but rare in unilateral tumors. J. Clin. Endocrinol. Metab. 87:1980-1985.

40. Weiss, J., and Zimmermann, F. 1999. Tribromoethanol (Avertin) as an anaesthetic in mice. Lab. Anim. 33:192-193.

41. Lavebratt, C.I., et al. 1998. Ovary transplantation method resulting in high reproductive performance in mice. Scand. J. Lab. Anim. Sci. 25:53-58.

42. Haavisto, A.M., et al. 1993. A supersensitive immunofluorometric assay for rat luteinizing hormone. Endocrinology. 132:1687-1691.

43. van Casteren, J.I., Schoonen, W.G., and Kloosterboer, H.J. 2000. Development of time-resolved immunofluorometric assays for rat follicle-stimulating hormone and luteinizing hormone and application on sera of cycling rats. Biol. Reprod. 62:886-894.

44. Bergendahl, M., Perheentupa, A., and Huhtaniemi, I. 1989. Effect of short-term starvation on reproductive hormone gene expression, secretion and receptor levels in male rats. J. Endocrinol. 121:409-417. 